\title{
Towards an RFID Microsystem for Surgical Instrument Detection Using Millimeter Waves
}

\author{
*M. Zamith, P.M. Mendes, Senior Member, IEEE \\ Departamento de Eletrónica Industrial \\ Universidade do Minho \\ Guimarães, Portugal \\ *a61745@alunos.uminho.pt
}

\begin{abstract}
Misplacement of surgical instruments inside an operating room is a frequent mistake that can bring unnecessary complications. We propose a system that can prevent these, through wireless communication. In this paper, we investigate the use of the 55-60 GHz band in the implementation of a RFID system that presents very interesting characteristics like improved security and small size. From the measurements already made, it's expected the RFID system operating in the $60 \mathrm{GHz}$ ISM will work at distances, at least, up to 5 meters. Furthermore, it is proposed a solution to obtain a fully integrated system based on an on-chip antenna that could be integrated with an RF CMOS chip based on $65 \mathrm{~nm}$ CMOS technology.
\end{abstract}

Index Terms - RFID microsystem, milimeter waves, $55 \mathrm{GHz}$, surgical misplacement

\section{INTRODUCTION}

Medical sponges and other surgical instruments are commonly misplaced or miscounted in the inside of an operating room (OR). In fact, a study from the National Center for Health Statistics found that an error of this type occurs in about one to every 5500 surgical procedures performed in the United States of America, which ads up to about twice a year in the average hospital [1].

Current practice to avoid these mistakes is to hand-count the surgical instrumentation before and after the procedure to find out if there are any missing items. However, this strategy can present several flaws associated with human mistake. Firstly, visual search takes up very expensive operating room time and trying to find a misplaced item could easily disrupt the hospital's surgical schedule. Also, as aforementioned, this methodology is very ineffective as errors occur at a relatively high rate, since surgical objects have been left behind in incisions of all sizes during nearly every type of surgery or invasive procedure. These errors occur more frequently during surgeries to the abdomen, pelvis and chest as well as during childbirth and gynecological procedures [2].

Serious problems can happen if objects are left behind inside patients' bodies, as the problem is only discovered when the patient complains of an adverse reaction. Victims not only suffer pain, but also a variety of additional consequences such as internal bleeding, blockages and infection.
Automatic detection of these items could not only prevent serious injuries and unnecessary follow-up procedures, but also reduce the number of malpractice claims related to the health service.

Some strategies are already under investigation and even on the market that offer a possible solution to the presented problem, like Image Guided Navigation Systems from Medtronic and BrainLAB [3]. However, these are expensive and take up a lot of room inside an operating room.

In this paper, we propose a solution associated with wireless communication through radiofrequencies. The operating principle behind a Radio Frequency Identification (RFID) system is fairly simple and extensively used in commercial applications such as security and supply chain management. The basic technology is presented in Figure 1 and it includes two components: one transponder (for each component that is to be identified) and a reader, which will interpret the data.

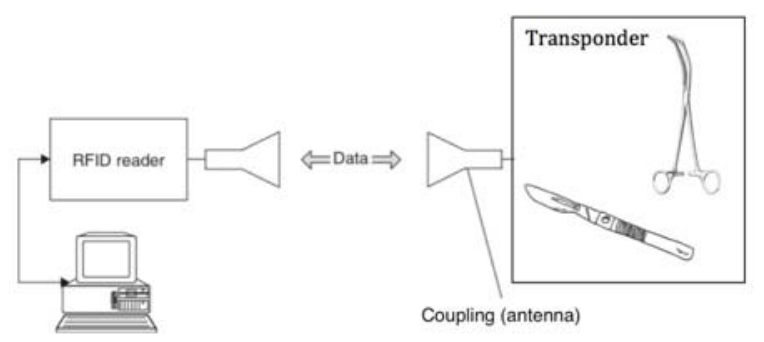

Figure 1. The reader and transponder are the two main components of the RFID system. The transponder will be placed in each surgical instrument (adapted from [4]).

In this case, the reader will most likely be a read only device. The tag, or transponder, will receive a signal from the reader and transmit it's unique ID number back to the reader. The reader contains a radio frequency module, a control unit and a coupling element to the transponder, which represents the actual data-carrying device of the RFID system.

This paper investigates the possibility of using a wireless link in the millimeter band to develop an RFID system that can be suitable to detect and track surgical tools. 
The paper is organized as follows: in section two, we justify the importance of the investigation and use of the $60 \mathrm{GHz}$ ISM band to complete the wireless link between components; in section 3, it's presented a simple design of the RFID system along with a brief explanation of the steps involved and respective challenges; in section 4, we show some experimental results already achieved regarding a wireless link at $58 \mathrm{GHz}$; in section 5, we speak about future improvements to the system, mainly regarding its miniaturization.

\section{RFID USING THE $60 \mathrm{GHz}$ ISM BAND}

RFID integrated systems working at HF and UHF bands have been widely implemented, namely in [5 - 8]. However, we chose to investigate the $55-65 \mathrm{GHz}$ band $(V$-band) due to its very unique characteristics. Millimeter Wave Identification (MMID) updates the RFID system to millimeter waves [9]. There are several advantages of MMID over RFID, like the wide bandwidth, which is useful for the transfer of big amounts of data (reaching gigabytes). This allows the design of transponders for high data rate applications as shown in [10]. However, for this specific application, we are more interested in one other significant advantage provided by the higher carrier frequency: the physical size of antennas at millimeterwave frequencies becomes so small that it becomes practical to build complex antenna arrays and further integrate them on chip or PCB $[11,12,13]$.

In this paper we propose to use MMID since smaller antennas enable miniaturized transponders [5], and compact reader modules. This means that ultra-small and light devices suitable for surgical tools are allowed by this wavelength. Also, improved security can be achieved due to high absorption.

\section{MMID SYSTEM DESIGN}

The block diagram of the system under study for data transmission is presented in Figure 2. In this section, every component is described with detail, as we try to pinpoint why every decision was made.

\section{A. Control Unit}

In the implemented test solution, the control unit is a microcontroller. Its purpose, in the transponder, is to generate the desired binary sequence that will serve as the unique ID of each one of the surgical tools. Since this system does not require a high data rate, it was selected an Atmega324 with an internal clock of $1 \mathrm{MHz}$, and $C$ language was used for programing. The reader's control unit will read and interpret the binary sequence that was previously sent by the transmitter. It will tell the user weather the ID corresponds to an expected sequence or not.

\section{B. Transmission}

As aforementioned, the control unit is responsible for coding the IDs and it does so using a NRZ procedure (a binary one is represented as "high" and a binary zero is represented as "low") [14]. When switching ON and OFF, the output voltage of the microcontroller can be directly used as the tune voltage of a voltage-controlled oscillator (ZX95-2150VW by minicircuits) to modulate the carrier signal for the desired frequency.
In the millimeter wave level, this process can be seen as an frequency modulation (frequency shift keying, FSK), since the end result will be a binary sequence that changes the carrier between two frequencies, when it switches ON and OFF the VCO tune voltage.

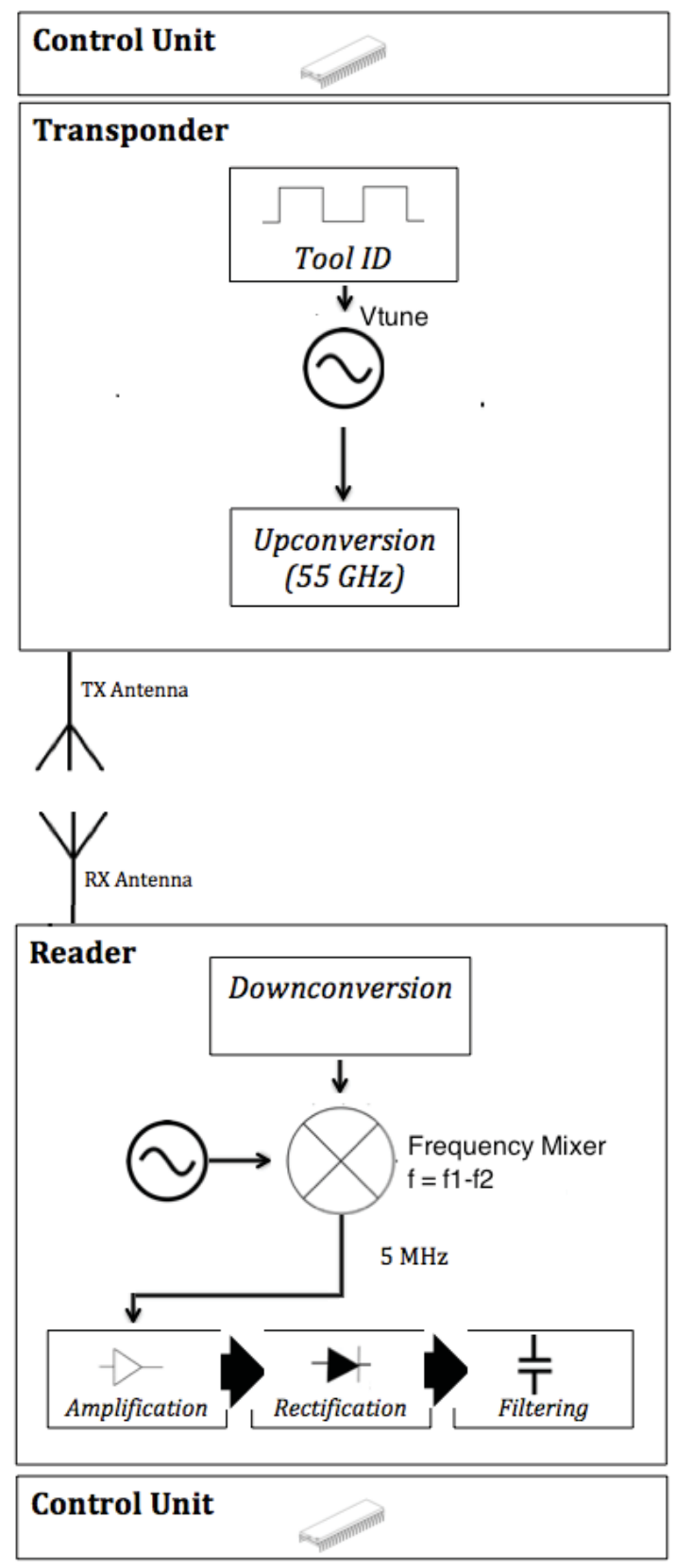

Figure 2. Block diagram of the designed system.

If a binary one is to be sent, the TX input intermediate frequency for upconversion will be higher than if a binary zero is to be transmitted. Table 1 indicates specific measured values through a spectrum analyzer (Agilent E4404B). 
The output from the VCO will be then upconverted so the transmission can occur at the desired carrier frequency In the $60 \mathrm{GHz}$ ISM band.

TABLE I. OUTPUT FREQUENCY OF THE VCO ACCORDING TO "HIGH" AND "LOW" VALUES OF TUNE VOLTAGE

\begin{tabular}{|c|c|}
\hline Tune Voltage (V) & Frequency (GHz) \\
\hline 0 (low) & 0,804 \\
\hline 5 (high) & 1,156 \\
\hline
\end{tabular}

\section{Reception}

Obtaining the desired binary signal after transmission requires RF to DC conversion. The first step of the proposed design, after acquisition of the output RX intermediate frequency is a second downconvesion to a frequency of about $5 \mathrm{MHz}$. This will facilitate the process of rectifying the signal.

To accomplish such task, a frequency mixer is used. The input signals are the output from the first downconversion and the output from a VCO with a carefully selected tune voltage, so that a signal with a frequency differing only $5 \mathrm{MHz}$ from the "high" frequency from Table 1 is generated at its output.

The output of the RF frequency mixer (ZFM-15 from minicircuits) is a signal whose frequency is the subtraction of the input signals' frequencies. Therefore, if the signal is "high" the resulting signal will have a frequency close to $5 \mathrm{MHz}$, however, if it's "low", the resulting frequency will be much higher.

After amplification, the signal is rectified and the higher frequencies are removed as a consequence of low-pass filtering. A DC proportional to the amplitude of the wave is the obtained.

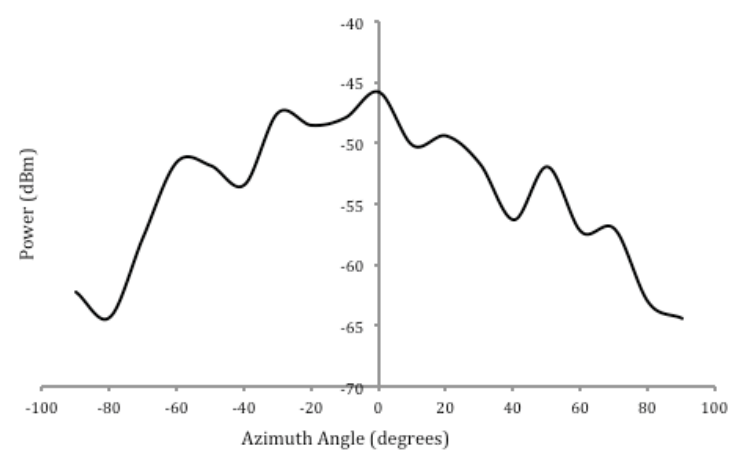

Figure 3. Output RX intermediate frequency power for several different rotation angles.

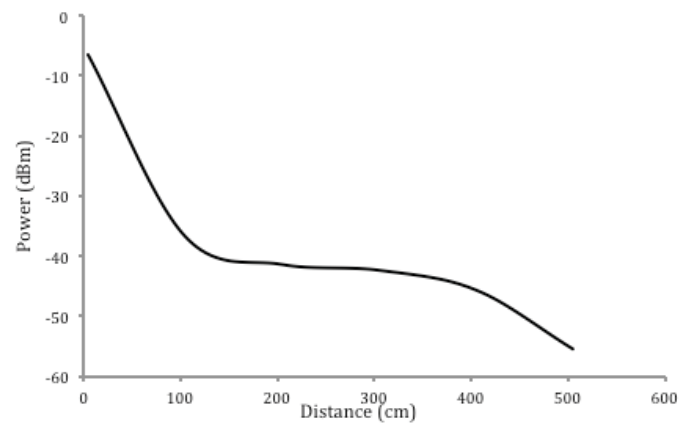

Figure 4. Output RX intermediate frequency power for several different distances between reciever and transmitter.

\section{FEASIBILITY EXPERIMENTS}

Due to the high path loss at the proposed frequencies, one's main issue is the potential range of the system. To assess the feasibility of the proposed solution for RFID applications, the

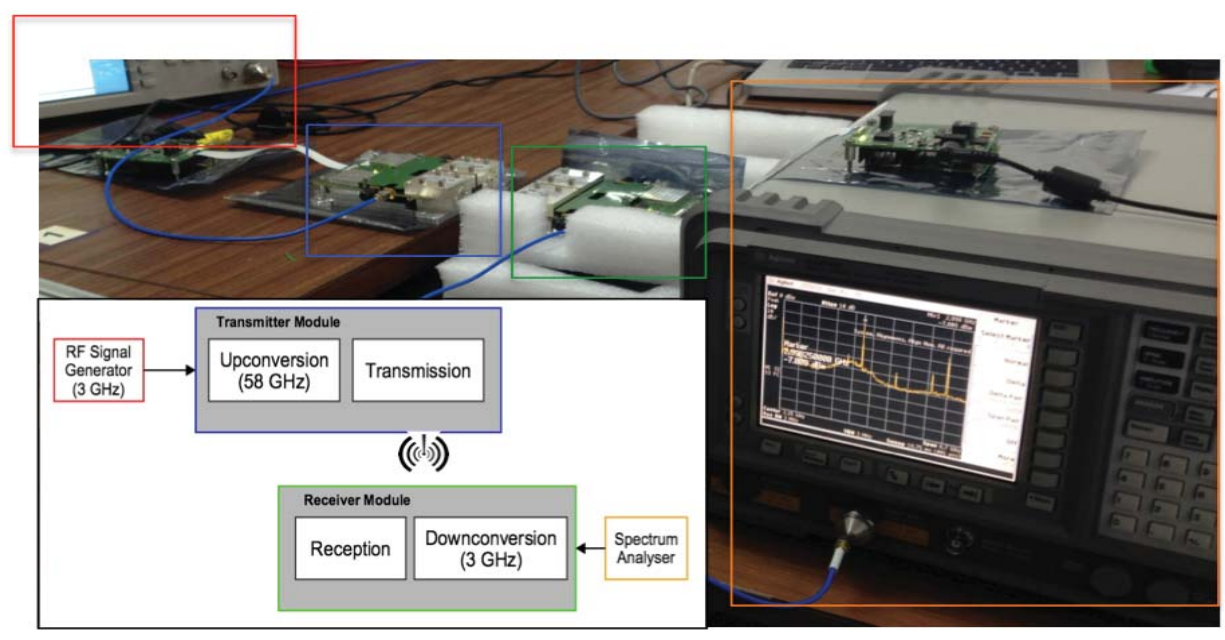

Figure 5. Setup used to implement the link at $58 \mathrm{GHz}$, as well as the respective block diagram. Color codes are used to match the block diagram to the picture. 
setup shown in Fig. 5 was used to implement a link in the $58 \mathrm{GHz}$.

\section{A. Implementation of a $58 \mathrm{GHz}$ Link}

The setup implemented to assess system range and performance was based on the V-band converter with LO, by Siversima ${ }^{\circledR}$. The input IR I-signal frequency of $3 \mathrm{GHz}$ was used with power of $-20 \mathrm{dBm}$. No IF Q-signal was used.

The used transceiver modules do not allow a direct measure of the received power, only at the IF frequency $(1-5 \mathrm{GHz})$, but since all the transceiver settings are kept constant during measurements, the power measured at the IF frequency attend as an indirect measurement of the expected attenuation when using such frequencies. To obtain data close to what will be obtained in a real scenario, the measurements were done inside the laboratory, without any particular attention to multipath, fading, or reflections.

The graphs represented in Figures 3 and 4 show the power of the output RX intermediate frequency at several different distances (figure 3) and angles (figure 4). To test the effect of the rotation of the device, a distance of 4 meters was fixed between transmitter and receiver.

We can safely conclude through the analysis of these figures that transmission was successfully achieved at a frequency of $58 \mathrm{GHz}$, namely at distances up 5 meters. We can also claim that the rotation of the device is influential, although the signal can still be detected when there is a misalignment between transmitter and receiver.

\section{Microsystem}

As mentioned in the previous sections, the main goal behind our focus in millimeter wave communication is the development of ultra-small devices.

As so, the final system under development is expected to include a chip implemented in a $65 \mathrm{~nm}$ technology, as per suggested in [15]. These authors successfully designed and implemented an array of distributed oscillators for millimeter wave phased arrays, which combines LO generation and distribution. The designed chip has an area of $6 \mathrm{~mm}^{2}$.

Figure 6 shows a representation of the proposed antenna integration solution for the on-chip antenna array proposed in [12].

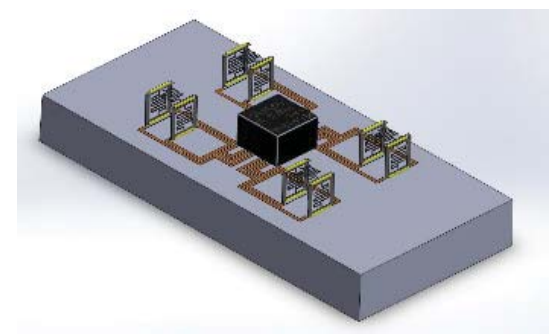

Figure 6. Represention of the proposed antenna integration solution [12]].

\section{CONCLUSIONS}

The $60 \mathrm{GHz}$ ISM is a frequency band that has still many possibilities to explore and that offers several advantages, namely in the fabrication of ultra-small footprint devices. The RFID microsystem under development shows promise in the detection of instruments at distances up to 5 meters.

Nevertheless, further experimentation and testing is required to continue developing the proposed application, so that it can accomplish its desired purpose, which is a system with the smallest possible dimensions, allowing placement in surgical tools, that are precision instruments. The final system is expected to have an area of $6 \mathrm{~mm}^{2}$.

\section{ACKNOWLEDGMENT}

This work was supported by Portuguese Foundation for Science and Technology: FCT-PTDC/EEI-TEL/2881/2012, Programa Operacional Temático Fatores de Competitividade (COMPETE) and Fundo Comunitário Europeu FEDER.

\section{REFERENCES}

[1] Linnan \& Fallon. Retained object surgical errors persist despite precautions. http://www.linnanfallon.com/Articles/Retained-objectsurgical-errors-persist-despite-precautions.shtml (accessed 15 February 2015).

[2] Tavss Fletcher. Medical Complications of a Retained Surgical Object. http://www.tavss.com/blog/common-injuries-of-misplaced-surgicaltools-within-the-body.cfm (accessed 15 Februray 2015).

[3] medGadget. Image Guided Spine Navigation Systems Improve Spine Surgeon's Screw Placement http://www.medgadget.com/2008/12/image guided spine navigation s ystems_improve_spine_surgeons_screw_placement.html (accessed 15 February 2015)

[4] Funkenzeller K. RFID Handbook. Third. Wiley; 2010

[5] Curty JP, Joehl N, Dehollain C, Declercq MJ. Remotely powered addressable UHF RFID integrated system. IEEE J Solid-State Circuits. 2005;40(11):2193-202.

[6] Yao Y, Wu J, Shi Y, Dai FF. A Fully Integrated 900-MHz Passive RFID Transponder Front End With Novel Zero-Threshold RF - DC Rectifier. IEEE Trans Ind Electron. 2009;56(7):2317-25.

[7] Glidden R, Bockorick C, Cooper S, Diorio C, Dressler D, Hara D, et al Design of ulra-low soct UHF RFID tags for supply chain applications. IEEE Commun Mag. 2004;42(8):140-51.

[8] Barnett R, Balachandran G, Lazar S, Kramer B, Konnail G, Rajasekhar $\mathrm{S}$, et al. A Passive UHF RFID Transponder for EPC Gen 2 with $-14 \mathrm{dBm}$ Sensitivity in $0.13 \mu \mathrm{m}$ CMOS. ISSCC 2007. 2007;582-4.

[9] Pursula P, Vähä-Heikkilä T, Müller A, Neculoiu D, Konstantinidis G, Oja A, et al. Millimeter-wave identification - A new short-range radio system for low-power high data-rate applications. IEEE Trans Microw Theory Tech. 2008;56(10):2221-8.

[10] Kiuru T, Pursula P, Rajamäki J, Vähä-heikkilä T. A 60-GHz Semipassive MMID Transponder for Backscattering Communications. 2013;9-11.

[11] $\mathrm{Yu} \mathrm{Y}$, Baltus PGM, Roermund AHM van. Integrated $60 \mathrm{GHz} \mathrm{RF}$ Beamforming in CMOS. Springer; 2011.

[12] Zamith M, Magalhães J, Anacleto P, Mendes PM. 60 GHz On-Chip Antenna Array with Efficiency Improvement Using 3D Microfabrication Technology. Accepted for the 9th European Conference on Antennas and Propagation (EuCAP). Lisbon. 2015.

[13] Zamith M, Dinis H, Magalhães J, Fernandes J, Mendes PM. On-Chip , Efficient and Small Antenna Array for Millimeter-Wave Applications. Accepted for the International Workshop on Antenna Technology 2015 (iWAT2015). Seoul, Republic of Korea. 2015.

[14] Pursula P, Donzelli F. Transponders for Millimeter Wave Identification. IEEE APS Top Conf Antennas Propag Wirel Commun. 2011;1221-4.

[15] Moroni A, Genesi R, Manstretta D. A Distributed "Hybrid "Wave Oscillator Array for millimeter-wave phased-arrays. Cust Integr Circuits Conf (CICC), 2012 IEEE. 2012;(1):1-4. 